\title{
Análisis de la calidad percibida en usuarios/as de servicios sociales comunitarios
}

\author{
Manuel E. Medina Tornero
}

Facultad de Trabajo Social, Universidad de Murcia

<memedina@um.es>

\section{Elvira Medina Ruiz}

Facultad de Trabajo Social, Universidad de Murcia

Erabiltzaileak zerbitzuan jasotako asebetetzea garrantzizko gaia da, eta kalitate-mailaren neurritzat ulertu izaten da. Horrela, gaiaren ikerketa bultzatzearen garrantzia nabarmentzen da erabiltzailearen behar eta nahietara moldarazteko. SERVQUAL eskala erabiliz, oinarrizko ongizate zerbitzuetako bi zentroetako erabiltzaileek hautemandako asebetetzea eta kalitatearen neurrien araberako aldeak aztertzen dira testuan. Gehienbat balioesten dute profesionalek transmititutako segurtasuna eta konfiantzaren arloa, eta puntuzenbatze baxuena jaso du erantzun ahal izateko gaitasunak, hau da, erabiltzaileen eskaeren aurrean profesionalen artean ematen diren zailtasunegoerak. 7 puntuko mailakatze batean 6,21 puntu jasotzen dira asebetetze-indizea aztertzean, eta, oro har, datua ontzat hartu daiteke bestelako zerbitzuen asebetetze-mailarekin erkatuz gero.

\section{HITZ-GAKOAK:}

kalitatea, hautemandako kalitatea, gizartezerbitzuak, ebaluazioa, SERVQUAL.
La satisfacción de las y los usuarios con la atención recibida se concibe como una medida de control de calidad, y su estudio es importante para conseguir una mejor adaptación a las necesidades y deseos de las y los usuarios. Utilizando la escala SERVQUAL, aquí se analizan algunos aspectos de medida de satisfacción y calidad percibida de los y las usuarias de dos centros de servicios sociales de base. El ámbito más valorado es el de la seguridad y la confianza que transmiten los profesionales en el trato, mientras que el aspecto que presenta menor puntuación es el de la capacidad de respuesta, entendida como la dificultad que tienen los profesionales para hacer frente a las demandas de las y los usuarios. El índice general de satisfacción alcanza 6,21 puntos en una escala de 7 , un buen dato si lo comparamos con otros análisis de satisfacción en prestación de servicios.

\section{Palabras Clave:}

calidad, calidad percibida, servicios sociales, evaluación, SERVQUAL. 


\section{Introducción}

En los últimos treinta años, se han producido importantes cambios sociales, culturales, económicos y tecnológicos que han afectado notablemente nuestra forma de vida y distintos ámbitos de la sociedad, entre ellos la forma en que los ciudadanos perciben las prestaciones de los servicios sociales. También podemos admitir que la población se ha vuelto más exigente hacia lo público, y no es de extrañar que demande prestaciones y considere que deben ser de calidad. Analizando con una cierta perspectiva histórica este proceso, puede afirmarse, sin dudarlo, que se han introducido cambios significativos en el número y la calidad de los recursos disponibles, y también que se han abordado cambios estructurales para satisfacer las nuevas demandas ciudadanas. La importancia que se atribuye ahora a la mejora de la calidad, sin ser nueva, es seguramente una de las transformaciones más llamativas.

Ahora bien, en el proceloso y difícil contexto de los servicios sociales, la calidad es, como señalan algunos teóricos y profesionales que la implementan, el fruto de una sabia receta en la que intervienen perspectivas cualitativamente diferentes, donde aproximar necesidades y expectativas a recursos y oferta sigue siendo la clave, aunque no siempre resulte fácil descifrarla. Es por ello por lo que la satisfacción del usuario o usuaria se ha abierto un hueco entre las medidas de resultado de las políticas sociales y, más concretamente, de los servicios sociales. Puede afirmarse, de este modo, que la puesta en marcha de métodos y canales para escuchar la voz de la persona usuaria constituye una revolución silenciosa, que paulatinamente irá introduciendo un cambio en la cultura de las organizaciones sociales.

La evaluación de las prestaciones y los servicios sociales por parte de las y los usuarios responde a las nuevas exigencias que deben reunir los servicios sociales, tal y como quedan reflejadas en las nuevas leyes de servicios sociales que se han ido aprobando en los últimos años, pero también al compromiso ético tradicional en las disciplinas de la intervención social. No obstante, adoptar modelos de gestión de calidad en ayuntamientos (en ciertos servicios o en su totalidad) implica ir más allá. Significa que la gestión en las organizaciones se hace por algo y, sobre todo, para alguien. Significa que prima la orientación hacia el cliente o usuario/a. Y es aquí, precisamente, donde se demuestra la validez y eficacia del despliegue de la calidad en las instituciones sociales.

\section{Contexto de la calidad}

\subsection{En busca de una definición de calidad}

Un planteamiento acerca de la calidad en las entidades prestadoras de servicios sociales parece que no pueda empezar de otro modo que ofreciendo una definición de qué se entiende por calidad. Pese a la dificultad que ello comporta y, hasta cierto punto, de la arbitrariedad de ofrecer cualquier definición, hemos creído conveniente 'enfrentarnos' a esta tarea, con el objeto de clarificar si existen elementos y argumentos constantes en el intento de definir qué es calidad.

Al término calidad le ocurre lo que a tantos otros a los que su amplia difusión ha terminado por desdibujar su significado, atribuyéndoseles tantos matices que los han convertido en expresiones que encierran sentidos diversos. La etimología remite al vocablo latino qualitas -atis. Nuestro diccionario de la Real Academia Española (1992) define calidad como "la propiedad o conjunto de propiedades inherentes a una cosa, que permiten apreciarla como igual, mejor o peor que las restantes de su especie". El mismo diccionario la define, también, en sentido absoluto, como "superioridad o excelencia".

Pero el enfoque actual de la calidad excede de la idea de un producto o servicio bien hecho o prestado. Tendiendo hacia un enfoque integrador de la gestión, la calidad total se concibe como la mejora continua de la gestión y de los resultados de la organización, de forma que se obtengan productos o servicios que den plena satisfacción al cliente, al menor coste económico posible, y con la participación e implicación de todo el personal de aquélla. Se trata, pues, de una mejora continua de la gestión y de los resultados, de buscar la satisfacción del cliente, y de conseguir la participación y responsabilidad de todos los miembros de la organización.

Según esto, los principios de la calidad total son:

- Mejora continua.

- Satisfacción del cliente.

- Compromiso de la dirección.

- Adhesión del personal.

- Eficiencia económica.

Así pues, la calidad puede ser vista como un resultado, una idea o un valor:

- La calidad es el resultado satisfactorio que se consigue cuando los productos o servicios se organizan y ofrecen de acuerdo con las necesidades y expectativas de los clientes, tanto externos como internos.

- Una idea amplia de calidad la encontramos en Morris (1992): “La calidad se alcanza cuando los servicios que se prestan, según los compromisos adquiridos, se adecuan a las necesidades reales de sus destinatarios y de la comunidad en general, y todo ello se hace de manera económica, efectiva, eficiente y equitativa".

- La calidad muestra el valor de las personas en el desempeño de sus tareas, su satisfacción con el trabajo bien hecho, siempre a la primera y con el empleo de los recursos estrictamente necesarios. 
Analizando las definiciones dadas en obras de carácter general, más que en documentos especializados, hallamos concepciones que pueden agruparse en función del elemento que priorizan, como 'la calidad en el diseño o de las características intrínsecas'; 'la calidad en el proceso o de la calidad como conjunto de maneras de proceder o de hacer'; y ‘la calidad centrada en el resultado o de la satisfacción de los clientes', siendo esta última la que nos interesa para este trabajo.

Existe en la actualidad sobrada evidencia empírica como para reconocer que el modelo de gestión de los centros públicos que opera en nuestro país, sean éstos del ámbito que sean, está sumido en una crisis, cuya influencia sobre la concepción de la gestión de calidad, aunque reclamada y conocida por los expertos su inexistencia, pasa a menudo inadvertida para la sociedad, entre otras razones, por la peculiar naturaleza del servicio que se proporciona -buena parte de cuyos efectos se advierten, principalmente, a medio y largo plazo- y por la escasa referencia a los resultados - a la medida de la eficiencia- y a la comparación, con fines evaluativos, que dicho sector presenta.

\subsection{Calidad de servicio}

La calidad de servicio es una variable de naturaleza compleja si lo pensamos desde la perspectiva que nos ofrece el análisis de sus características específicas. Esta complejidad aumenta si nos referimos a los servicios sociales, pues la prestación del servicio y su consumo, en unas ocasiones se producen simultáneamente, y en otras se retrasa, o nunca llega a producirse. De ahí que se deba estar atento a las emociones y sensaciones que se producen durante su prestación, ya que, a diferencia de otros servicios, son muy importantes para la implicación del profesional con las personas usuarias demandantes.

La conceptualización de la calidad de servicio ha sido un debate que ha suscitado un especial interés. Inicialmente se entendía desde una definición centrada en la realización de un servicio de acuerdo con las especificaciones técnicas fijadas por los prestatarios, abordándose el tema como si de un producto se tratara. Posteriormente, la definición empezó a desplazarse hacia el usuario o usuaria, viéndose desde la perspectiva subjetiva de éste. De esta manera, se pasó de una concepción más técnica y objetiva a otra más subjetiva y centrada en los requerimientos del usuario o usuaria. A partir de esta concepción última (la subjetiva), aparece el término calidad percibida frente al de calidad técnica (Carman, 1990). Sin embargo, Grönroos (1994) ya aportó la idea de que los y las usuarias comparan sus expectativas con el servicio que perciben que han recibido, siendo el resultado de este proceso la calidad percibida del servicio. Si se introduce la percepción, la definición pasa a entenderse desde la óptica del usuario o usuaria; por lo tanto, la única referencia de calidad real es la que existe en la mente de éste/a.

En la literatura sobre calidad de servicio, está ampliamente aceptada la concepción de que la calidad de servicio percibida depende de la comparación del servicio esperado con el servicio percibido y es, por esta razón, el resultado de un proceso de evaluación continuado. Es decir, se trata del resultado de la comparación evaluativa que el usuario hace de la experiencia percibida del servicio con las expectativas que tenía en el momento de la compra o el consumo. Esta definición se adapta al denominado paradigma no confirmatorio aplicado a la satisfacción, el cual establece que la satisfacción se relaciona con el tamaño y la dirección de una experiencia no confirmatoria, donde dicha experiencia es la diferencia entre las expectativas iniciales del individuo y el resultado actual del servicio. Así pues, las expectativas de un usuario/cliente son:

- Confirmadas cuando un servicio conforma las expectativas.

- Negativamente confirmadas cuando el servicio no resulta como se esperaba.

- Positivamente confirmadas cuando el servicio resulta mejor de lo esperado.

\section{Satisfacción del usuario/a}

La satisfacción ciudadana por la calidad de un servicio está relacionada con el conjunto de propiedades que debe tener ese servicio para atender, en primer lugar, las necesidades (explícitas e implícitas) de las personas a las cuales va destinado. Pero esto, aunque sea necesario, no es suficiente, porque la satisfacción va ligada también a la percepción que el ciudadano tiene de cómo se presta el servicio y a la expectativa que tenía respecto al servicio esperado. Esta situación nos lleva a un lugar conflictivo, como es la concepción del ciudadano como cliente de los servicios, y esto sí significa un enorme cambio en las bases políticas de las administraciones, especialmente la municipal. Como bien indica García Roca (1992: 31), “el Estado no se justifica por el respeto a las leyes, la división de poderes y los procedimientos de la toma de decisiones, sino por las prestaciones del Estado que exigen los ciudadanos". Las especiales características de las personas que son receptoras de servicios sociales han conducido a ciertos autores e investigadores a establecer una distinción funcional entre consumidor, usuario/a y cliente.

Para este estudio, nos interesa de forma especial el concepto de usuario/a que hace referencia al ciudadano contribuyente que usa el servicio que le ofrece la Administración -a través de funcionarios o empresas concertadas- de forma gratuita y en igualdad de condiciones. En estas circunstancias, el usuario o usuaria no suele ejercer el derecho a exigir calidad en la prestación. En el modelo de servicio público, cuentan más los intereses de los funciona- 
rios que las expectativas y necesidades de las y los usuarios, quienes normalmente no tienen plataformas de presión eficaces. Ball (1997) llama a este modelo burocorporativo.

Corresponde que nos preguntemos en este momento: ¿qué significa satisfacción del usuario o usuaria? Sobre este criterio se fundamenta gran parte de la filosofía de las organizaciones que muestran algún interés por la calidad. Es tan importante que se convierte en el núcleo de toda la actividad de la organización. Las instituciones que deciden trabajar con criterios de calidad, utilizando alguno de los modelos existentes, planifican, generan procesos, diagnostican, se autoevalúan, toman decisiones, producen servicios, crean estructuras y mecanismos de comunicación para el cliente y en función de éste. Por esta razón, es de gran importancia que podamos definir de forma operativa lo que significa satisfacción del usuario o usuaria. Siguiendo algunas de las reflexiones de Álvarez Fernández (1998), destacamos los dos puntos que tienen gran utilidad para nuestro argumento:

- Identificar los factores críticos de éxito que constituyen el núcleo de las expectativas del usuario o usuaria. Para ello, es fundamental conocer (escuchar) las necesidades y demandas de los y las usuarias, y solicitar su opinión sobre la satisfacción del servicio.

- Destacar la importancia del personal de primera línea (trabajador social, psicólogo, educador), que son las personas claves en el proceso interventivo, de manera especial, cuando surge una situación de conflicto o insatisfacción con el usuario o usuaria: para ello, es necesario conocer el mayor número de aspectos de estos profesionales, con el fin de calibrar el papel que auténticamente desempeñan en la prestación de los servicios.

Por lo tanto, el objetivo de cualquier estudio que desee determinar la percepción de calidad de los y las usuarias de un centro de servicios sociales deberá contemplar, además de los ámbitos ya considerados de cultura organizacional y clima, aspectos y variables actitudinales, como el nivel de satisfacción laboral, el burnout y el compromiso organizacional que presentan los profesionales, puesto que son las personas con mayor contacto con los y las usuarias y, como consecuencia, las responsables de influir sobre la percepción de calidad asistencial que éstos/as puedan tener.

\subsection{Medida de la satisfacción del usuario o usuaria en servicios sociales}

La satisfacción de las y los usuarios con la atención recibida es concebida como una medida de control de calidad, por lo que su estudio y valoración es importante para gestión y el trabajo de los profesionales de los servicios sociales en su conjunto, con la perspectiva de conseguir una mejor adaptación a las necesidades y deseos de las y los usuarios. Autores como Vuori (1982), Suñol (1987), Medina Tornero (1996) o Saturno (1991), además de organizaciones como la OMS, la Unesco y la Federación Española de Municipios y Provincias (Brezmes, 2010), han señalado la pertinencia de introducir la satisfacción de los y las usuarias con los servicios como parte y complemento de otras actividades de control de calidad. Desde nuestra perspectiva, el objetivo principal de conocer la satisfacción de los y las usuarias es obtener datos para llevar a cabo una intervención que permita mejorar las actuaciones profesionales.

La satisfacción del usuario o usuaria en relación al resultado de la asistencia, es decir, su opinión acerca de si el resultado obtenido era el mejor posible, no solía estar incluida en las medidas de satisfacción (Pallares y García, 1996). En las evaluaciones de calidad asistencial sanitaria, tales juicios los realizan normalmente los profesionales, porque no es probable que los pacientes tengan los conocimientos técnicos necesarios para emitir un juicio sobre los máximos beneficios alcanzables por la asistencia sanitaria. Todo esto ha cambiado lo suficiente, llegando, incluso, a la creación de organizaciones de consumidores que asesoran a los pacientes, y de otro tipo de organizaciones de defensa del consumidor que reclaman la participación del paciente en todo lo que tiene que ver con el proceso asistencial (Martínez del Olmo, 1994; Gómez y Saturno, 1997). Sin embargo, los y las usuarias del sistema de servicios sociales sí tienen criterio en muchas ocasiones, incluso los conocimientos necesarios para mantener una opinión definida (Medina Tornero, 1997a; 1997b).

Lo cierto del caso es que algunas medidas de satisfacción en términos de calidad percibida de los servicios se obtienen comparando las percepciones con las expectativas, con ciertas diferencias sutiles. La satisfacción compara las percepciones de los consumidores con lo que normalmente esperarían, mientras que la calidad percibida de los servicios compara las percepciones de los consumidores con lo que un consumidor debería esperar de una empresa que ofrece servicios de elevada calidad; es decir, tal como argumentan Hoffman y Bateson (2002: 324), "la calidad de los servicios parece medir un parámetro más alto de la prestación del servicio".

En este sentido, la calidad percibida de los servicios, por lo general, es más compleja de evaluar que las de los productos, principalmente las características distintivas (intangibilidad, variabilidad, caducidad, y que son producidos y consumidos simultáneamente), aunque éstas pueden variar con mayor o menor intensidad de un empleado a otro, o de un cliente a otro. Por consiguiente, diversos investigadores, entre ellos Hoffman y Bateson (2002), concuerdan en afirmar que la calidad de los servicios es un concepto escurridizo y abstracto, difícil de definir y medir; es por esta razón por la que se le ha dado importancia a la estandarización de los servicios para proveer una calidad consistente. 


\subsection{Calidad de servicio percibida}

Prestigiosos investigadores han estudiado científicamente la calidad de servicio percibida desde diferentes puntos de vista, manteniendo un debate interesante sobre el concepto, la dimensión y la medida de la calidad de servicio. En este apartado, se revisan de forma somera los principales modelos que han ayudado al desarrollo de este constructo (Medina Tornero, 2000).

\subsubsection{El modelo de Parasuraman, Zeithaml y Berry}

La calidad de servicio recibió un fuerte impulso para su aplicación en la gestión de las organizaciones a partir de los trabajos desarrollados, hace años, por Parasuraman, Zeithaml y Berry $(1985,1988,1993$, 1994), cuyo modelo es un punto de referencia obligado en el estudio y la gestión de la calidad.

Específicamente $(1985,1988)$, definen la calidad de servicio percibida como el juicio global del cliente acerca de la excelencia o superioridad del servicio, que resulta de la comparación entre las expectativas de los consumidores (lo que ellos creen que las empresas de servicios deben ofrecer) y sus percepciones sobre los resultados del servicio ofrecido. Indican que, a la hora de evaluar la calidad de servicio, y en ausencia de criterios de carácter objetivo, es necesario llevar a cabo un diagnóstico a partir del análisis de las percepciones de los o las clientes o usuarios/as con relación al servicio recibido (Parasuraman, Zeithaml y Berry, 1988). Por ello, la calidad del servicio queda definida, en este modelo, como la amplitud de las diferencias o brechas que existe entre las expectativas o deseos de los clientes y sus percepciones respecto a la prestación del servicio (Parasuraman, Zeithmal y Berry, 1993).

Teniendo en cuenta las conclusiones alcanzadas en sus investigaciones, estos autores desarrollaron un modelo conceptual de calidad del servicio y diseñaron un instrumento de medida, que identificaron como SERVQUAL (SERVice QUALity), para su evaluación (Parasuraman, Zeithaml y Berry, 1988). El SERVQUAL se fundamenta en la teoría de los gaps (the Gaps models of service quality), la cual explica las diferencias entre las expectativas de los clientes y aquello que realmente obtienen del servicio utilizado (Igami, 2005). El estudio exploratorio de estos investigadores ha sido considerado una innovación dentro del área de evaluación de servicios.

El modelo SERVQUAL se fundamenta en la premisa de que todas las usuarias y usuarios de servicios poseen una expectativa de calidad del servicio que se les oferta. La diferencia entre la expectativa y la percepción se denomina gap (falla), y en ella reside la oportunidad para la mejoría del servicio (Igami, 2005). Después de años de refinamiento de su escala, el SERVQUAL utiliza actualmente cinco dimensiones de abordaje, destinadas a medir la diferencia entre la expectativa del usuario o usuaria y la satisfacción con el servicio.

\subsubsection{El modelo de Grönroos}

En su modelo, Grönroos (1988) define la calidad de servicio percibida conectando las experiencias en calidad con las expectativas en calidad. El autor entiende que las expectativas son función de una serie de factores, tales como la comunicación de mercado, la comunicación boca a oído, la imagen corporativa/local y las necesidades del cliente.

\subsubsection{El modelo de Nguyen}

Nguyen (1991) se basa en el sistema de servucción ${ }^{1}$ para determinar su modelo, que se define a partir de las relaciones entre la imagen de la empresa, el resultado del personal en contacto con el cliente, la organización interna de la servucción, el soporte físico de la servucción, y la satisfacción del cliente. Así pues, según este autor, cuanto mayor sea la percepción del cliente en cada una de estas variables, mayor será su percepción de la calidad del servicio. Por tanto, las variables anteriores son consideradas como factores explicativos de la variable dependiente.

\subsubsection{El modelo de Bolton y Drew}

Bolton y Drew (1991a) presentan la calidad de servicio percibida como la resultante de la satisfacción/ insatisfacción a través de la no confirmación de expectativas, mediante las percepciones de niveles de resultado de los componentes. De su investigación, deriva que un determinante clave de la calidad de servicio global es el desajuste entre resultado y expectativas (Bolton y Drew, 1991b). Entienden que la calidad de servicio es una actitud frente a la empresa y todos sus servicios, mientras que la satisfacción se expresa con respecto al servicio ofrecido personalmente. También concluyen diciendo que es de la calidad de servicio percibida de la que se derivan las intenciones comportamentales.

\subsubsection{El modelo de Bitner}

Bitner (1990) define la calidad de servicio percibida como una consecuencia de la experiencia de satisfacción/insatisfacción. La autora indica que el

\footnotetext{
${ }^{1}$ De una manera sencilla, puede definirse la servucción como el proceso de elaboración de un servicio, es decir, toda la organización de los elementos físicos y humanos en la relación cliente-empresa que es necesaria para prestar de un servicio, y cuyas características han sido determinadas - desde la idea concebida hasta la obtención del resultado- como tal servicio. Resumiendo, podríamos decir que es todo el engranaje necesario que hay detrás de la prestación de un buen servicio. El usuario/cliente no ve la servucción, sino su resul-
} tado. 
paradigma no confirmatorio se da entre el resultado del servicio percibido en una transacción y las expectativas iniciales. Esto afecta a la experiencia de satisfacción/insatisfacción y, posteriormente, a la calidad de servicio percibida, de la cual se deriva la comunicación boca a oído, el cambio de servicio y la fidelidad.

\subsubsection{El modelo de Koelemeijer, Roest y Verhallen}

Koelemeijer, Roest y Verhallen (1983). Estos autores aportan un modelo con una estructura integradora, en la que el resultado y las expectativas se encuentran en tres planos:

- Macro: expectativas y percepciones de resultado respecto a clase-productos.

- Meso: expectativas y percepciones de resultado con respecto al proveedor de servicio.

- Micro: expectativas y resultado percibidos con respecto a una transacción individual.

\subsubsection{El modelo de Grönroos-Gummesson}

El modelo de Grönroos-Gummesson (Gummerson, 1987, cit. por Grönroos, 1994) se propone ayudar tanto a los fabricantes como a las empresas de servicios en la gestión de la calidad. Se basa en dos modelos con dos enfoques separados sobre la descripción de cómo se crea la calidad: a) el modelo 4 Q de Gummesson, basado en la noción de que todo el mundo contribuye a la calidad y de que existen diferentes fuentes de la calidad en una empresa; y b) el modelo de Grönroos de la calidad percibida en los servicios, que trata de la percepción de la calidad. Estos autores proponen cuatro fuentes de la calidad: el diseño, la producción, la entrega y las relaciones. La forma de gestionar y resolver estos aspectos afecta a la calidad percibida por el cliente. Tanto la calidad técnica del resultado de los bienes o servicios como la calidad funcional de los procesos interactivos que implican al comprador y al vendedor están influidas por estas fuentes de la calidad.

\subsection{La calidad y la satisfacción en la administración pública}

Como se ha podido observar, la calidad de servicio y la satisfacción son conceptos muy estudiados en la literatura del marketing. Este desarrollo de la investigación se ha centrado principalmente en el sector privado de la economía. En cambio, el sector público ha sido el que, hasta la fecha, menos ha podido beneficiarse de este tipo de estudios, debido sobre todo a la escasa sensibilidad de sus responsables por conocer las necesidades y expectativas de los ciudadanos.
Como afirma Gaona (2001), la implantación de la gestión de la calidad total en la administración pública, a través de cualquiera de los modelos existentes, suscita reacciones encontradas. Por una parte, se encuentran aquellos que entienden que implantar uno de estos sistemas será la solución a multitud de circunstancias y vaivenes en el servicio público. Y por otra parte se encuentra el escepticismo hacia estos sistemas y procesos de implantación de la calidad total en el sector público. Desde esta última perspectiva, se entiende que en la administración pública, la implantación del modelo de calidad europeo (EFQM) puede resultar un fracaso, debido a la idiosincrasia de las administraciones y de las relaciones que se establecen entre éstas y los ciudadanos. Tanto las acepciones de la calidad en la administración pública como las barreras a las que deben enfrentarse están, en parte, relacionadas con la evolución que ha sufrido, y aún está sufriendo, el modelo de administración pública.

Senlle (1996) indica que los sistemas de gestión de la calidad deben implantarse en la administración pública, no ya por conseguir votos, sino por converger con los países más avanzados de Europa, y aporta diez razones concretas:

- Para asegurar el futuro y la continuidad del equipo.

- Por economía y para reducir el déficit.

- Para mejorar el servicio al cliente interno.

- Para mejorar el servicio al cliente externo.

- Para integrar el cliente a la organización.

- Por mejorar la imagen.

- Para ser más competitivos.

- Para mejorar la gestión.

- Para obtener resultados en los objetivos y la economía.

- Para potenciar el estilo de liderazgo.

En definitiva, la administración pública está adquiriendo cada vez más responsabilidad en su atención al ciudadano, y fruto de ello son las numerosas iniciativas que se están desarrollando para implantar sistemas de certificación, control o de gestión de calidad. En definitiva, se trata de un lento proceso de modernización de las administraciones que se presenta como una necesidad urgente para los ciudadanos a los que sirve. Consecuentemente, las administraciones se enfrentan con el reto de conciliar la satisfacción de los derechos individuales con la atención a los intereses colectivos, de promover la cohesión social y de ejecutar las políticas públicas. Ello justamente en un periodo histórico en el que la globalización y la proliferación de entramados transnacionales de personas y de organizaciones trasladan a la Administración responsabilidades acrecentadas. 


\subsection{Situación actual del análisis de satisfacción en los servicios de base}

Son escasos los servicios sociales que elaboran de manera sistemática estudios de opinión de la satisfacción de las personas que los utilizan. En los casos en los que se realizan, se utilizan cuestionarios, metodologías y enfoques dispares que hacen muy difícil realizar comparaciones y agregar los resultados. Se trata, pues, de un ámbito en el que hay un amplio margen para el desarrollo. La experiencia en materia de evaluación de la satisfacción de las personas usuarias de los servicios sociales va adquiriendo relevancia en los últimos años. En el ámbito de los servicios sociales básicos, se han desarrollado diferentes experiencias de evaluación de la satisfacción de las personas usuarias ${ }^{2}$, aunque hemos de admitir que no con carácter empírico, en su mayoría.

Pero hemos de admitir que, aunque se hagan encuestas de satisfacción a los y las usuarias de servicios sociales, su trascendencia social es escasa, y prueba de ello es la ausencia de referencias en la literatura científica que las avalen o que, simplemente, sirvan para que se conozca su existencia, a diferencia de lo que ocurre en el campo sanitario, en el que hay cientos de artículos sobre análisis de satisfacción de pacientes y sobre aspectos de la calidad percibida, tanto en la sanidad comunitaria como en la hospitalaria.

En el campo general de los servicios municipales, existen algunas referencias publicadas sobre satisfacción percibida de las y los usuarios, por ejemplo, de instalaciones deportivas (Vila, Sánchez y Manassero, 2009; Morales Sánchez, 2003; Gálvez Ruiz y Morales Sánchez, 2011), recogida de residuos (Pérez Morote, 2001) o servicios socioculturales (Catalina, Palacios y Fulgueiras, 2002).

\section{El marco de la calidad en las administraciones públicas}

Cuando se traslada el concepto de calidad al ámbito de las administraciones públicas, no hay que olvidar que, en ellas, concurren una serie de circunstancias que deben tenerse en cuenta, principalmente la enorme variedad de sectores de la vida ciudadana en los que está presente la Administración y, en consecuencia, el elevado número y la gran heterogeneidad de los servicios prestados. Por ello, hablar de calidad

\footnotetext{
${ }^{2}$ En particular, nos referimos a: el estudio Servicios sociales y satisfacción del Ayuntamiento de Reus (2003), que se ha tenido en cuenta para elaborar el plan de calidad de los servicios sociales de ese municipio (2009); las encuestas de satisfacción entre personas usuarias de servicios de atención domiciliaria realizadas en el periodo 2002-2005 en diferentes municipios de Barcelona, con el apoyo de la Diputación de Barcelona; el estudio que la Fundación EDE realizó en el País Vasco sobre la implantación de sistemas de calidad en los servicios locales de la CAPV (2008), y que se refiere la realización de una serie de encuestas a las y los usuarios de servicios sociales; el trabajo de carácter metodológico de Cabrera et al. (2010) sobre la satisfacción de usuarios y usuarias de programas sociales; y la investigación de Sánchez Pérez (2010) sobre la mejora de los servicios sociales.
}

en las administraciones públicas significa, sobre todo, hablar de un nuevo enfoque en su actuación, considerando al ciudadano como cliente, consumidor individual de los servicios prestados.

La calidad debe constituirse en el nexo de unión que garantiza la coherencia de toda la actividad de la organización. Por un lado, trata de conseguir un funcionamiento armónico de la organización (coordinado y que consigue los resultados deseados) y, por otro, debe establecer las bases para la mejora, para la gestión del cambio y la continua adaptación de la organización al entorno. Para ello, aporta diversidad de herramientas y metodologías de trabajo. Por otro lado, la calidad se construye con un liderazgo político y profesional del más alto nivel, plenamente convencido de la necesidad de cambio y comprometido en ello, sobre cinco ejes básicos:

- La satisfacción de la ciudadanía: la prestación del servicio ha de estar claramente orientada al usuario o usuaria, conocer las demandas de la ciudadanía y sus prioridades, evaluar sus expectativas y su satisfacción con el servicio recibido, e incorporar estas opiniones en la planificación y el rediseño del servicio, para su mejora continua.

- Objetivos y compromiso de resultados ante la ciudadanía: deben planificarse las acciones para satisfacer aquellas necesidades, articulando objetivos a corto y largo plazo, explicitando claramente los compromisos que se adquieren ante la ciudadanía en cuanto a resultados en la prestación de servicios y cumpliendo los objetivos con el menor coste posible. Es importante el establecimiento de indicadores, la medición y la evaluación de resultados.

- Los procesos de producción de los servicios: deben identificarse y estudiarse los procesos de gestión de los diferentes servicios, conocerse sus costes, los tiempos de entrega del servicio, la productividad y la posición en relación con los otros municipios (los del entorno y los mejores).

- La satisfacción de las personas que trabajan en la Administración: hay que ser conscientes de la importancia de las personas y de lo necesario de la mejora continua en términos de satisfacción y compromiso. Debe explicitarse claramente a las personas del Ayuntamiento hacia dónde se quiere ir y con qué propósito, lo que da sentido al trabajo de los empleados y, por tanto, aumenta su motivación y compromiso.

- La participación de todos los grupos de interés en la mejora continua y toma de decisiones.

Se define, en consecuencia, la calidad total y la mejora continua como el marco más idóneo para el inicio de una intervención normalizadora y simplificadora de los procesos.

Es evidente que la puesta en marcha de sistemas de gestión de calidad está avanzando en las administraciones españolas, pero estamos aún lejos de poder 
hablar de su generalización. Como suele suceder, hay un grupo de administraciones que lideran la implantación de estos sistemas y son tomadas como referentes por los demás.

Con la publicación, en 1992, de la Ley de Régimen Jurídico de las Administraciones Públicas y del Procedimiento Administrativo Común, se produce un cambio en las relaciones de la Administración con la ciudadanía, al reconocérsele más derechos, hacerse más sencillos los procedimientos e instaurarse el uso de las tecnologías de la información, dentro de la perspectiva de la Administración como entidad prestadora de servicios.

Todos los niveles de la Administración española han realizado un gran esfuerzo por modernizarse y han desarrollado acciones diversas que obedecen, sin embargo, a objetivos comunes, entre los que se pueden destacar: acercar la Administración al ciudadano; mejorar la atención y el servicio prestado, implantando sistemas de calidad y midiendo la satisfacción de ciudadanía y usuarios/as; o lograr una Administración ágil y accesible. A este respecto, es necesario poner de manifiesto que implantar sistemas de gestión de calidad constituye un elemento más dentro de los procesos de modernización en que están incursas la administración general del Estado, las comunidades autónomas y una gran mayoría de entidades locales; unos procesos de modernización basados en la combinación de tres elementos básicos: la simplificación, la información y la tecnología.

\subsection{La calidad de los servicios sociales en las administraciones públicas}

La introducción, en el Sistema para la Autonomía y Atención a la Dependencia (SAAD), de la calidad como concepto y como mecanismo articulador que favorezca el control y la eficacia ha sido, sin duda, uno de los grandes aciertos de la denostada, $y$ al mismo tiempo esperada, Ley 39/20063. Pero ha tenido un efecto multiplicador, ha conseguido que nos interesemos por la aplicación práctica de la calidad y ha logrado que las leyes posteriores incluyeran la calidad como referencia y marco operativo (Medina Tornero y Medina Ruiz, 2011).

El desarrollo de los distintos estatutos de autonomía ha hecho que las comunidades autónomas sean las responsables de otorgar las correspondientes autorizaciones, así como de crear y actualizar los registros de centros y servicios, lo que ha facilitado el cumplimiento de los requisitos y estándares de calidad. También son las comunidades autónomas quienes establecen el régimen jurídico y las condiciones de actuación de los centros privados concertados, además de acreditar a los centros y servicios privados no concertados que prestan servicios a personas mayores en situación de dependencia. Con el fin de

${ }^{3}$ Ley de Promoción de la Autonomía Personal y Atención a la Dependencia. asegurar la calidad de esa atención, se gestiona y asegura la correcta acreditación de centros, y se definen criterios y parámetros como requisitos mínimos para obtener dicha acreditación. En estos momentos, algunas comunidades autónomas están llegando más lejos y han comenzado a elaborar planes de calidad, planes estratégicos y similares.

A partir de nuevas leyes y actuaciones, las comunidades autónomas quieren extender y sistematizar los desarrollos en materia de calidad a las prestaciones que ofrecen los sistemas públicos, con el fin de promover un proceso de mejora continua en la calidad que pueda ser evaluado objetivamente, tanto interna como externamente. Estos planes (se denominen de calidad, de servicios sociales, geriátricos o estratégicos), al igual que los requisitos para recibir la autorización y la acreditación, se han establecido de forma independiente por parte de cada comunidad autónoma, sin que haya un mínimo común. Esta falta de criterios comunes ha dado lugar a que, en cada comunidad, se hayan dado diferentes desarrollos, con distintos grados de complejidad y, por lo tanto, sea difícil establecer un estándar nacional.

Aquí utilizaremos, como marco referencial, la Ley Catalana de Servicios Sociales (Ley 12/2007, de 11 de octubre), que contempla, en el título VII, todo lo concerniente a la calidad y su operatividad en servicios sociales. La calidad viene definida en la ley desde dos perspectivas: por un lado, como principio rector de todo el Sistema Catalán de Servicios Sociales, y por otro, como un derecho de las personas usuarias en su relación con el sistema. Más adelante, se le dedica un capítulo, en el que se establece que el plan de calidad es la herramienta básica para asegurar el desarrollo y la aplicación de los principios de calidad, y se concreta que incluirá "los estudios de opinión y los resultados de los procedimientos de participación de los usuarios y de sus familias" (artículo 85.3c). Por otra parte, en el artículo 10e) de la misma ley, se establece que las personas tienen derecho a "presentar sugerencias, obtener información, poder presentar quejas y reclamaciones, $\mathrm{y}$ recibir respuesta dentro del periodo legalmente establecido". La ley ha dado lugar a un plan de calidad, en el que la calidad de servicio se define por el nivel de satisfacción que las personas usuarias muestran respecto a la actuación de los servicios sociales, particularmente respecto a la satisfacción de sus necesidades y expectativas (pág. 21-25). En consecuencia, la apreciación de la calidad del servicio corresponde a las personas a las que se dirige 0 , en un sentido más amplio, al conjunto de personas o entidades interesadas o afectadas, en una $u$ otra medida, por este servicio (grupos de interés).

El primer eje estratégico del plan de calidad es la mejora de la calidad de las prestaciones en su integridad, incluyendo tanto el proceso de acceso a la prestación como el de seguimiento una vez ésta ha sido concedida. Y el primer objetivo estratégico es, precisamente, el de evaluar la satisfacción de las personas usuarias de las prestaciones garantizadas provistas por el sistema público. 
Pero también debemos contemplar la calidad desde la óptica de los ayuntamientos, que son los últimos responsables de las prestaciones de los servicios sociales comunitarios. Aquí debemos hacer referencia a los esfuerzos de la Federación Española de Municipios y Provincias de aproximar la calidad a los ayuntamientos y de concienciar de su importancia en los procesos de modernización de las entidades locales (Brezmes, 2010). Es de resaltar que también contamos con la experiencia de la trayectoria de gestión de la calidad de un gran número de entidades locales ${ }^{4}$. De gran valor orientativo y documental es el trabajo realizado por la Fundación EDE en el País Vasco entre 2007 y 2009, impulsado por el Gobierno Vasco y con la colaboración de Euskalit, al objeto de fomentar la implantación de la calidad en los ayuntamientos vascos 5 .

En el sistema de servicios sociales, la tendencia parece imparable. La planificación y la gestión de la calidad se van convirtiendo en una exigencia para la contratación y concertación de servicios con la empresa privada. AENOR ya hace tiempo que estructuró la certificación de calidad para las organizaciones de servicios sociales en la Familia de Normas UNE 158000, a partir de unos requisitos mínimos y consensuados ${ }^{6}$ :

- UNE 158101: gestión de centros residenciales con centro de día o centro de noche.

- UNE 158201: gestión de centros de día y de noche.

- UNE 158301: gestión del servicio de ayuda a domicilio.

- UNE 158401: gestión del servicio de teleasistencia.

En 2005, se presentó una adaptación específica de la norma ISO a los Gobiernos locales (ISO IWA 4), cuyos ejes son:

- Desarrollo institucional para un buen gobierno7.

- Desarrollo ambiental sustentable.

- Desarrollo económico sostenible.

- Desarrollo social incluyente.

4 Véanse referencias en Escobar Rando y Gil Valenzuela (2004); Sánchez Aguado (2011); Sacanell (2003); Moya Vaquero (2000); Cercadillo (2008); Del Pozo Herráiz y Sánchez Pérez (2010).

${ }^{5}$ Véase una aproximación en Barbero y Díez Aramburu (2009).

${ }^{6}$ Véase una experiencia de gran interés en Espinosa (2007).

7 El documento IWA 4 proporciona lineamientos para la aplicación de la norma ISO 9001:2000 en los Gobiernos locales, describe ejemplos puntuales que facilitan la interpretación y, además, cuenta con un mapa de procesos que proporciona una guía para organizar la gestión de los Gobiernos locales. Incluye también una herramienta integral de autodiagnóstico, que contiene parámetros de medición de los elementos mínimos que un Gobierno local no puede dejar de tener, y que enriquece la aplicación de las directrices. El IWA 4 tiene como objetivo establecer las directrices para auxiliar al Gobierno local en la implementación de un sistema de gestión de la calidad que cumpla los requisitos de la norma ISO 9001:2000, de tal manera que se logren condiciones confiables de calidad en las actividades que realiza el Gobierno local para satisfacer los requisitos de la población presente en su territorio.
Hoy podemos afirmar, sin miedo a equivocarnos, que la mejora de la calidad de los servicios públicos y, en particular, de los servicios sociales municipales, equivale a la defensa del sistema público de servicios sociales. También podríamos preguntarnos por qué es tan importante la calidad en los servicios sociales comunitarios, de base o de atención primaria. Parafraseando a Ángel Parreño (2007), podríamos indicar lo siguiente:

- Por la estructura de los equipos de atención primaria: complejos, con roles profesionales en constante redefinición, con problemas de estabilidad en la plantilla, con vaivenes políticos y voluble definición de programas y prestaciones.

- Por las características de la toma de decisiones: alta autonomía en la atención y la prescripción profesional, puerta de entrada a un sistema que frecuentemente cierra la siguiente puerta (servicios especializados, con límites definidos e inflexibles), decisiones interinstitucionales.

- Por las dificultades de la vida municipal (resistencias internas a simplificar y agilizar procedimientos), y la tendencia permanente al fraccionamiento de competencias y la creación de nuevos 'chiringuitos'.

- Por la variabilidad de modelos de funcionamiento y de cobertura de necesidades.

Nosotros añadiríamos un motivo más: porque son el rostro que reconocen las y los usuarios, y es preciso que se midan con la necesaria cautela las relaciones que se establecen, de forma que se proceda al análisis de satisfacción de las y los usuarios y se les devuelva la información, con el fin de que sean partícipes del proceso prestacional (Medina Ruiz, 2011).

\section{Metodología}

\subsection{Objetivos}

Esta investigación tiene como objetivo principal evaluar la calidad percibida por los y las usuarias de dos centros de servicios sociales del Ayuntamiento de Murcia, mediante el instrumento SERVQUAL. Para alcanzar este objetivo general, se han propuesto los siguientes objetivos específicos:

- Identificar las principales dimensiones de calidad, según el instrumento SERVQUAL.

- Conocer la opinión que tienen las y los usuarios sobre las dimensiones de calidad.

- Analizar y elaborar conclusiones.

\subsection{Población objetivo}

La recogida de información se ha realizado durante los meses de mayo a julio de 2010. La población objeto del estudio ha sido el conjunto de usuarias y 
usuarios del Centro de Servicios Sociales 'Ciudad de Murcia' y del Centro de Acción Comunitaria 'La Paz-La Fama'. La muestra comprende un total de 41 individuos (hombres y mujeres) que utilizan el servicio de atención primaria de dichos centros. Para llevar a cabo la recogida de datos, nos hemos desplazado a los centros, y a las personas que habían visitado el servicio de atención primaria les hemos ofrecido la oportunidad de responder voluntariamente al cuestionario, mediante una entrevista.

\subsection{Instrumento de análisis}

Hemos utilizado un cuestionario basado en el modelo propuesto por Parasuraman, Zeithmal y Berry (1988) para medir variables tales como la tangibilidad, la fiabilidad, la capacidad de respuesta, la profesionalidad, la cortesía, la credibilidad, la seguridad y la accesibilidad. El presente estudio sólo ha medido la percepción del servicio de atención primaria, debido a que la mayoría de las personas encuestadas eran asiduos a estos servicios, y poseían una confirmación (o no) de sus expectativas, por lo que no se podía medir el gap entre las expectativas y las percepciones del servicio prestado. La Escala SERVQUAL tiene 32 ítems medida y es una escala Likert de 7 puntos que van desde 'totalmente de acuerdo' (7) hasta 'nada de acuerdo' (1). En este caso, el conjunto de las preguntas han sido formuladas de forma positiva, por lo que no ha habido que invertir valores. La administración del cuestionario, con formato de entrevista, se realizó durante los días de atención en las unidades de trabajo social mencionadas. Para el tratamiento y análisis de los datos, se utilizó el paquete informático SPSS 15.0 para Windows.

\section{Análisis de resultados}

\subsection{Datos sociodemográficos}

A continuación presentamos los datos sociodemográficos generales, referidos a las personas que han participado en el estudio, centrándonos en las variables relativas a sexo, edad, estado civil, nivel de estudios y situación laboral.

Como hemos dicho anteriormente, el estudio, se realizó en dos centros de servicios sociales del Ayuntamiento de Murcia. En el centro A, participaron 18 sujetos, y en el centro B, 24. El 59,5\% (seis de cada diez) eran mujeres, proporción desigual que pone de manifiesto el marcado predominio de las mujeres entre los usuarios y usuarias de servicios sociales. Mayoritariamente se encontraban casados o solteros (70\% de la muestra), seguidos de los viudos (19\%), y los separados y divorciados ( $4,8 \%)$.

Por segmentos de edad, el grupo más numeroso es el comprendido entre 51 y 60 años (33,3\%), seguido por el de 31 a 40 años (23,8\%). En cuanto al nivel de estudios, el grupo mayoritario es el de estudios básicos o primarios, con un $42,9 \%$ de la muestra. Se observa un importante número de encuestados sin estudios ( $26,2 \%$ ), siendo más acentuada esa tendencia entre las mujeres que entre los hombres. Otro dato destacable es la ausencia de encuestados con titulación universitaria, ya sea media o superior. No debemos olvidar el progresivo envejecimiento de la población usuaria, así como su feminización y bajo nivel educativo.

En cuanto a la situación laboral de los encuestados, observamos cómo los dos grupos mayoritarios (que representan más de la mitad de la muestra) están formados por desempleados (31\%) y 'otros' (31\%), categoría compuesta principalmente por jubilados y pensionistas. Le siguen los empleados por cuenta ajena $(26,2 \%)$, las amas y los amos de casa $(9,4 \%)$ y los autónomos (2,4\%). Sobresale la ausencia de funcionarios. El resultado está relacionado con las altas tasas de paro que predominan actualmente y con el envejecimiento de la población usuaria, que pertenece generalmente al cupo de 'otros'.

\subsection{Dimensiones de calidad percibida}

Una vez analizadas las variables sociodemográficas que conforman la muestra, se procede a comentar los resultados que miden la calidad percibida del servicio de atención primaria de los dos centros de servicios sociales que han sido objeto de estudio. Recordamos que el instrumento elegido para ello ha sido la escala SERVQUAL. Con él medimos las distintas dimensiones de la calidad percibida del servicio, que se agrupan en cinco bloques (Tabla 1).

Tabla 1. Resultado de las dimensiones de calidad percibida

\begin{tabular}{l|l|c}
\hline Dimensión & Descripción & $\begin{array}{c}\text { Puntuación } \\
\text { media }\end{array}$ \\
\hline $\begin{array}{l}\text { Elementos } \\
\text { tangibles }\end{array}$ & $\begin{array}{l}\text { Apariencia de las } \\
\text { instalaciones, equipo, } \\
\text { personal y materiales } \\
\text { para comunicaciones }\end{array}$ & 6,05 \\
\hline Confiabilidad & $\begin{array}{l}\text { Capacidad para brindar } \\
\text { el servicio prometido en } \\
\text { forma precisa y digna de } \\
\text { confianza }\end{array}$ & 6,39 \\
\hline $\begin{array}{l}\text { Capacidad de } \\
\text { respuesta }\end{array}$ & $\begin{array}{l}\text { Buena disposición para } \\
\text { ayudar a los clientes } \\
\text { a proporcionarles un } \\
\text { servicio expedito }\end{array}$ & 5,73 \\
\hline Seguridad & $\begin{array}{l}\text { Conocimiento y cortesía } \\
\text { de los empleados, así } \\
\text { como capacidad para } \\
\text { transmitir seguridad y } \\
\text { confianza }\end{array}$ & 6,70 \\
\hline Empatía & $\begin{array}{l}\text { Cuidado y atención } \\
\text { individualizada que la } \\
\text { empresa proporciona a } \\
\text { su clientes }\end{array}$ & 6,32 \\
\hline
\end{tabular}

Fuente: Elaboración propia. 
Como observamos en la Tabla 2, los 'elementos tangibles' son los que peor puntuación obtienen en las valoraciones de las y los usuarios, con una media de 6,05. Dentro de éstos, se aprecian diferencias. Lo que mejor perciben es lo relacionado con el aspecto y aseo de los profesionales, que casi obtiene una puntuación de $7(6,98)$, seguido de las instalaciones agradables y atractivas $(6,00)$. Sin embargo, cuando se les pregunta por la modernidad del equipamiento y por los materiales relacionados con el centro, los encuestados los valoran con un 5,81 y un 5,43 respectivamente, unas puntuaciones de las más bajas de todo el cuestionario. El usuario o usuaria interactúa no sólo con el profesional, sino también con los aspectos tangibles asociados al servicio, que dan forma a sus impresiones acerca de la calidad del servicio del centro (Morales y Calderón, 2005).

Tabla 2. Resultado de la dimensión 'elementos tangibles'

\begin{tabular}{l|c|c|c}
\hline & Media & Desv. típ. & Media \\
\hline $\begin{array}{l}\text { Las instalaciones del centro } \\
\text { de servicios sociales son } \\
\text { agradables y atractivas }\end{array}$ & 6,00 & 1,40 & \\
\cline { 1 - 2 } $\begin{array}{l}\text { Los profesionales del } \\
\text { centro de servicios sociales } \\
\text { presentan buen aspecto y } \\
\text { van aseados }\end{array}$ & 6,98 & 0,15 & \\
\cline { 1 - 2 } $\begin{array}{l}\text { El centro de servicios } \\
\text { sociales cuenta con un } \\
\text { equipamiento moderno }\end{array}$ & 5,81 & 1,70 & \multirow{2}{*}{6,05} \\
\cline { 1 - 2 } $\begin{array}{l}\text { Los materiales } \\
\text { relacionados con el centro } \\
\text { (folletos explicativos, } \\
\text { web, cartelería...) son } \\
\text { visualmente atractivos }\end{array}$ & 5,43 & 1,64 & \\
\hline
\end{tabular}

Fuente: Elaboración propia.

En cuanto a la dimensión 'confianza' (Tabla 3), la puntuación media es superior a los elementos tangibles, con un 6,39. La variable que obtiene una mayor valoración es la referida a la manera 'clara y comprensible' de informar, que obtiene 6,86 , casi la máxima puntuación. Le siguen 'el personal del centro realiza bien el trabajo desde la primera vez' $(6,52)$, mantener el sistema de información y registro al día $(6,33)$, cumplir lo que se promete hacer en cierto tiempo $(6,19)$ y la seguridad de las instalaciones $(6,05)$. La dimensión 'confianza' significa que el servicio se brinda sin fallas (McElwee y Redman, 1993). Los usuarios y usuarias esperan que el servicio sea confiable $y$, por ello, puntúan muy positivamente que no haya errores (Prasuraman, Zeithaml y Berry, 1998).

La dimensión 'capacidad de respuesta' (Tabla 4) es la que obtiene la puntuación más baja de las cinco dimensiones, con un 5,73 de media. La dimensión mejor valorada es 'el personal me informa con precisión de cuándo concluirá el servicio ofrecido’ $(6,14)$, seguida de 'los profesionales se encuentran disponibles para atenderme' $(6,00)$. Realizar las actividades con prontitud y atender rápidamente a las peticiones es lo menos valorado, con un 5,71 y 5,05 respectiva- mente, siendo esta última la puntuación más baja de todo el cuestionario.

Tabla 3. Resultado de la dimensión 'confianza'

\begin{tabular}{|c|c|c|c|}
\hline & Media & Desv. típ. & Media \\
\hline $\begin{array}{l}\text { Cuando el personal del } \\
\text { centro de servicios sociales } \\
\text { promete hacer algo en } \\
\text { cierto tiempo, lo cumple }\end{array}$ & 6,19 & 1,06 & \multirow{5}{*}{6,39} \\
\hline $\begin{array}{l}\text { Las instalaciones del centro } \\
\text { de servicios sociales son } \\
\text { seguras }\end{array}$ & 6,05 & 1,78 & \\
\hline $\begin{array}{l}\text { El personal del centro de } \\
\text { servicios sociales realiza } \\
\text { bien su trabajo desde la } \\
\text { primera vez }\end{array}$ & 6,52 & 0,92 & \\
\hline $\begin{array}{l}\text { Los profesionales del centro } \\
\text { de servicios sociales me } \\
\text { informan de una manera } \\
\text { clara y comprensible }\end{array}$ & 6,86 & 0,35 & \\
\hline $\begin{array}{l}\text { Los profesionales del } \\
\text { centro de servicios sociales } \\
\text { mantienen el sistema de } \\
\text { información y registro al día }\end{array}$ & 6,33 & 1,22 & \\
\hline
\end{tabular}

Fuente: Elaboración propia.

Tabla 4. Resultado de la dimensión 'capacidad de respuesta'

\begin{tabular}{l|c|c|c}
\hline & Media & Desv. típ. & Media \\
\hline $\begin{array}{l}\text { El personal del centro de } \\
\text { servicios sociales no está } \\
\text { demasiado ocupado para } \\
\text { responder rápidamente a } \\
\text { mis peticiones }\end{array}$ & 5,05 & 1,62 & \\
\hline $\begin{array}{l}\text { El personal del centro } \\
\text { de servicios sociales me } \\
\text { informa con precisión de } \\
\text { cuándo concluirá el servicio } \\
\text { ofrecido }\end{array}$ & 6,14 & 1,12 & \\
\hline $\begin{array}{l}\text { Los profesionales del centro } \\
\text { de servicios sociales se } \\
\text { encuentran disponibles } \\
\text { para atenderme }\end{array}$ & 6,00 & 1,17 & \\
\hline $\begin{array}{l}\text { Los trabajos relacionados } \\
\text { con la acogida y } \\
\text { seguimiento, tales como } \\
\text { entrevistas, informes, } \\
\text { cuestionarios, etc., se } \\
\text { realizan con prontitud }\end{array}$ & 5,71 & 1,44 & \\
\hline
\end{tabular}

Fuente: Elaboración propia.

La dimensión 'seguridad' (Tabla 5) es la mejor valorada de todas, con una puntuación media de 6,70. Las variables que la componen están relacionadas con el trato recibido por parte del profesional (que obtiene la puntuación máxima, 6,95), la cualificación del profesional y su capacidad para inspirar confianza (6,76 cada una) y la recogida de forma adecuada de las sugerencias y quejas por parte de los profesionales $(6,33)$. Los resultados obtenidos coinciden con los publicados por Instituto Nacional de la Seguridad Social (Pérez Menayo, 2007), que se ha decantado por adoptar el modelo SERVQUAL para avanzar en su estrategia de calidad. En ese estudio, aparece que las 
usuarias y usuarios de los servicios sanitarios conceden una mayor importancia a la variable 'seguridad'.

Tabla 5. Resultado de la dimensión 'seguridad'

\begin{tabular}{l|c|c|c}
\hline & Media & Desv. típ. & Media \\
\hline $\begin{array}{l}\text { El comportamiento de los } \\
\text { profesionales del centro } \\
\text { de servicios sociales me } \\
\text { inspira confianza }\end{array}$ & 6,76 & 0,69 & \\
\hline $\begin{array}{l}\text { El personal del centro de } \\
\text { servicios sociales está } \\
\text { totalmente cualificado para } \\
\text { las tareas que realiza }\end{array}$ & 6,76 & 0,53 & \\
\hline $\begin{array}{l}\text { Los profesionales del centro } \\
\text { de servicios sociales se } \\
\text { muestran dispuestos a } \\
\text { ayudarme, y su trato es } \\
\text { considerado y amable }\end{array}$ & 6,95 & 0,22 & \multirow{2}{*}{6,70} \\
\cline { 1 - 2 } $\begin{array}{l}\text { Los profesionales del } \\
\text { centro de servicios sociales } \\
\text { recogen de forma adecuada } \\
\text { mis quejas y sugerencias } \\
\text { como usuario }\end{array}$ & 6,33 & 1,34 & \\
\hline
\end{tabular}

Fuente: Elaboración propia.

La dimensión 'empatía' (Tabla 6) obtiene una puntuación media de 6,32. Dentro de ella, se observan diferencias entre las variables encargadas de medir la calidad percibida. Lo mejor valorado es el trato personalizado $(6,57)$ y la comprensión, por parte de los profesionales, de las necesidades como usuario/a; pero si hablamos de cómo satisfacen los horarios las necesidades como usuario/a, la puntuación desciende $(5,76)$. Hay autores que apuntan que las bajas puntuación en la dimensión de empatía puede deberse a que el instrumento SERVQUAL podría no cubrir correctamente esta dimensión de la calidad en aquellos casos en los que la interacción profesionalusuario/a es muy intensa (Villegas y Rosa, 2003), como por ejemplo, en servicios sanitarios o de atención primaria en un centro de servicios sociales.

Dentro del cuestionario, se incluyó un ítem a modo de resumen, para que los encuestados hicieran una valoración general sobre el servicio prestado (Tabla 7). La puntuación media de dicha variable es de 6,21.
Tabla 6. Resultado de la dimensión 'empatía'

\begin{tabular}{l|c|c|c}
\hline & Media & Desv. típ. & Media \\
\hline $\begin{array}{l}\text { El personal del centro } \\
\text { de servicios sociales } \\
\text { me ha dado un trato } \\
\text { personalizado }\end{array}$ & 6,57 & 0,97 & \\
\hline $\begin{array}{l}\text { El centro de servicios } \\
\text { sociales tiene horarios } \\
\text { que satisfacen mis } \\
\text { necesidades como }\end{array}$ & 5,76 & 1,49 & \multirow{2}{*}{6,32} \\
$\begin{array}{l}\text { usuario } \\
\text { Los profesionales del } \\
\text { centro de servicios } \\
\text { sociales comprenden } \\
\text { cuáles son mis } \\
\text { necesidades }\end{array}$ & 6,62 & 0,96 & \\
\hline
\end{tabular}

Fuente: Elaboración propia.

Tabla 7. Satisfacción general

\begin{tabular}{l|c|c}
\hline & Media & Desv. típ. \\
\hline $\begin{array}{l}\text { Mis sentimientos hacia los servicios } \\
\text { que presta el centro de servicios } \\
\text { sociales pueden ser descritos como } \\
(1=\text { muy insatisfactorios, }\end{array}$ & 6,21 & 0,78 \\
$\begin{array}{l}7=\text { excelentes })\end{array}$ & & \\
\hline
\end{tabular}

Fuente: Elaboración propia.

Una vez analizado el resultado por variables de las dimensiones de calidad, se procede a relacionarlo con los factores sociodemográficos, con el fin de detectar posibles resultados significativos. En la valoración media de las dimensiones según el sexo, se ha determinado que no existen diferencias significativas entre hombres y mujeres. Sin embargo, a la hora de analizar las variables por distintos grupos de edad (Tabla 9), encontramos diferencias significativas en las dimensiones 'elementos tangibles' y 'empatía'. En elementos tangibles, las y los usuarios que más alto valoran son los del grupo de edad de 31 a 40 años $(6,40)$, seguidos de los de 51 a 60 años $(6,14)$, los de 21 a 30 y 41 a 50 (6,08 cada uno) y, por último, las y los usuarios de más de 60 años $(5,21)$. La diferencia entre el que más alto califica y el que menos es de 1,19. En la dimensión 'empatía', son los mayores de 60 años quienes mejor valoran las capacidades de los profesionales $(6,89)$, mientras que los que peor

Tabla 8. Valoración media, según grupos de edad

\begin{tabular}{l|c|c|c|c|c}
\hline Valor promedio & $\mathbf{2 1 - 3 0}$ años & $\mathbf{3 1 - 4 0}$ años & $\mathbf{4 1 - 5 0}$ años & $\mathbf{5 1 - 6 0}$ años & \multicolumn{1}{c}{$\mathbf{6 0}$ años } \\
\hline Elementos tangibles & 6,08 & 6,40 & 6,08 & 6,14 & 6,21 \\
\hline Confianza & 6,20 & 6,32 & 6,73 & 6,60 \\
\hline Capacidad de respuesta & 5,58 & 5,90 & 6,00 & 5,68 \\
\hline Seguridad & 6,92 & 6,38 & 6,88 & 6,71 \\
\hline Empatía & 6,56 & 6,33 & 6,22 & 6,03 \\
\hline Media total & 6,28 & 6,32 & 6,43 & 6,89 & 6,16 \\
\hline
\end{tabular}

Fuente: Elaboración propia. 
Tabla 9. Valor promedio, según estado civil

\begin{tabular}{l|c|c|c|c|c}
\hline Valor promedio & Soltero/a & Casado/a & Separado/a & Divorciado/a & Viudo/a \\
\hline Elementos tangibles & 6,28 & 6,08 & 7,00 & 6,00 & 5,80 \\
\hline Confianza & 6,41 & 6,31 & 6,80 & 6,55 \\
\hline Capacidad de respuesta & 5,80 & 5,50 & 6,50 & 7,00 \\
\hline Seguridad & 6,72 & 6,55 & 6,75 & 7,00 \\
\hline Empatía & 6,33 & 6,20 & 5,00 & 6,88 \\
\hline Media total & 6,35 & 6,14 & 6,51 & 6,67 \\
\hline
\end{tabular}

Fuente: Elaboración propia.

valoran son los de 51 a 60 años $(6,00)$. En este caso, la diferencia entre ambos es menor, de sólo 0,89. Para el resto de dimensiones, no hay diferencias significativas.

\section{Conclusiones}

Los resultados nos indican que, desde un punto de vista global, no existen grandes diferencias entre las distintas dimensiones de calidad percibida que hemos medido. Sin embargo, como primera apreciación, el análisis muestra tres resultados estrechamente vinculados y que, a efectos del estudio, son importantes: los de 'seguridad', 'confianza' y 'empatía'.

Las dimensiones que mejor han sido valoradas han sido las relacionadas con las capacidades del profesional: su disposición al trabajo, su cualificación, su capacidad para inspirar confianza o cumplir los plazos, entre otras. Las peor valoradas, por su parte, han sido las ajenas a la labor del profesional, los elementos tangibles, los referidos al aspecto y las funciones del centro, y a la capacidad de respuesta, que son aquellas variables que miden la rapidez con que se realiza el servicio, o la disponibilidad de los trabajadores, entre otras.

La primera conclusión que se puede derivar es que la percepción que las y los usuarios tienen de la labor profesional es buena o muy buena. Estos resultados no son novedosos si tenemos en cuenta que el analizado es un servicio de tipo asistencial, en el que el factor humano prima por encima de las instalaciones. En un servicio donde el contacto con el usuario o usuaria es tan elevado como lo es la relación establecida entre el usuario o usuaria y el trabajador social, es lógico que las variables referidas a la labor profesional cobren una mayor importancia. Esto sucede, por ejemplo, al medir la calidad percibida en las y los usuarios de un centro de salud (Villegas y Rosa, 2003).

Los y las usuarias califican por debajo de la media cuando se les pregunta por los elementos tangibles, excepto en la variable 'aspecto y aseo' de los profesionales. Los centros de servicios sociales objeto del estudio no son de nueva construcción, ni están dotados de un equipamiento moderno y visualmente atractivo enfocado a las y los usuarios, lo que conlleva que los usuarios no tengan una calidad percibida alta sobre ellos.

Que los servicios de atención primaria están desbordados es una realidad, y las y los usuarios así lo han manifestado: la dimensión con una calidad percibida más baja es la relacionada con realizar las tareas con prontitud, ya sea una cita por teléfono, una visita o una entrevista. Los profesionales, debido al colapso, no siempre se encuentran disponibles para atender de forma rápida las demandas de usuarias y usuarios. Tener un horario de atención tan constreñido también hace que las citas se demoren y que los y las usuarias tengan que esperar, a veces, hasta un mes, para ser atendidos por el trabajador social. La mayoría, además, contesta negativamente si le preguntan si el horario de atención satisfacía sus necesidades como usuario/a.

Nos encontramos ante un reto importante en los servicios sociales del Ayuntamiento de Murcia y el resto de ayuntamientos. Queremos que se abra paso un cambio en la cultura de los centros, para reorientarlos hacia las y los usuarios. La voz de éstos debe incorporarse al diseño y la evaluación de los procesos asistenciales para determinar el nivel de excelencia alcanzado. 
ÁLVAREZ FERNÁNDEZ, M. (1998): El liderazgo de la calidad total, Madrid, Editorial Escuela Española.

AYUNTAMIENTO DE REUS (2009): Pla de Qualitat dels Serveis Socials, Reus, Ayuntamiento de Reus [rhttp:// www.reus.cat/sites/default/files/Fitxers/ arees/benestar/documents/MvaLeNvUmA. $p d f>]$.

BALL, S. J. (1997): “La escuela participativa en el contexto de la política postmoderna y el market del modelo participativo en U.K”, en GARAGORRI, X., Participación, autonomía y dirección en los centros docentes, Madrid, Forum Europeo de Administradores de la Educación y Escuela Española.

BARBERO, I.; y DÍEZ ARAMBURU, V. (2009): “Orientaciones para la implantación progresiva de sistemas de calidad en los servicios sociales de la CAPV", Zerbitzuan, no-45, págs. 39-47 [ [http://www. zerbitzuan.net/documentos/zerbitzuan/3. pdf〉].

BITNER, M. (1990) "Evaluating service encounters: The effects of physical surroundings and employee responses", Journal of Marketing, n- 54 , págs. 69-82.

BOLTON, R.; y DREW, H. (1991a): “A longitudinal analysis of the impact of service changes on customer attitudes”, Journal of Marketing, $\mathrm{n}$ 55 , págs. 1-9 [<http://www.ida.liu.se/ steho/und/ htddo1/9602205187.pdf>].

- (1991b): “A multistage model of customers'assessments of service quality and value", Journal of Consumer Research, n- 17 , págs. 375-384 [fftp://ftp.cba.uri.edu/Classes/banerjee/ websyagnik/webpage/technology $\% 20$ adoption $\% 20$ and $\% 20$ lifestyles/service $\% 20$ \%20quality\%2ond\%2ovalue.pdf $)$.
BREZMES, Mำ J (2010): Propuesta para la configuración de un modelo actualizado de servicios sociales municipales, Madrid, Federación Española de Municipios y Provincias [<http://www.femp. es/files/566-894-archivo/nuevos_servicios_ sociales._libro.pdf〉].

CABRERA, F. A.; et al. (2010): "Valoración de la satisfacción de usuarios de programas sociales: propuesta de un modelo de análisis", Revista de Educación, no 351, págs. 311-336 [<http://www. revistaeducacion.mec.es/re351/re351_13.pdf)].

CARMAN, J. M. (1990): “Consumer perceptions of service quality: An assessment of the SERVQUAL dimensions", Journal of Retailing, $\mathrm{n}^{0}$ 66, págs. 33-55.

CATALINA, C.; PALACIOS, J. L; y FULGUEIRAS, F. J. (2002): “La medición de la calidad en la administración local: el caso de los servicios socioculturales del Ayuntamiento de Alcobendas", Dirección y Organización. Revista de Dirección, Organización y Administración de Empresas, no- 28, págs. 147-158.

CERCADILLO, M. (2008): “Un viaje hacia la calidad de los servicios sociales municipales: experiencia de una mancomunidad", Trabajo Social Hoy, nํ5 54, págs. 71-82.

DEL POZO HERRÁIZ, B.; y SÁNCHEZ PÉREZ, M. C. (2010) "Trabajando por la calidad desde los servicios sociales básicos”, Bits. Boletín Informativo Trabajo Social, no 14 [<http://www.uclm.es/ bits/sumario/79.asp〉].

ESCOBAR RANDO, G.; y GIL VALENZUELA, C. (2004): “El sistema de gestión de la calidad del Centro de Servicios Sociales Comunitarios del Distrito Centro: profesionalización y garantía de derechos", Documentos de Trabajo Social. 
Revista de Trabajo y Acción Social, nº 33 , págs. 55-114.

ESPINOSA, E. (2007): "Gestión de calidad en los servicios sociales. ISO 9001 y compromiso social", UNE. Boletín de AENOR, n- 217, págs. 22-25.

FUNDACIÓN EDE (2008) Proyecto de investigación-acción sobre la gestión de la calidad en el tercer sector de acción social de Bizkaia (20052008) / Bizkaiko gizarte ekintzako hirugarren sektoreko kalitatearen kudeaketaren inguruko ikerketa-ekintza proiektua (2005-2008), Bilbao, Fundación EDE [<http://www.fundacionede. org/calidad/docs/contenidos/_calidadCAST\%20\%2022\%20julio_.pdf〉, 〈http://www. fundacionede.org/calidad/docs/contenidos/_ PUBLICACION-calidad-EUSK $\% 20 \% 2022 \% 20$ julio_.pdf $\rangle$.

GÁLVEZ, P.; y MORALES, V. (2011): “Evaluación de la calidad en programas municipales deportivos: generalizabilidad y optimización de diseños de medida", Cuadernos de Psicología del Deporte, vol. 11, no extra 2, págs. 123-130.

GARCÍA ROCA, J. (1992): Público y privado en la acción social, Madrid, Editorial Popular.

GAONA, J. L. (2001): “Una herramienta para evaluar la calidad de los servicios públicos: la aplicación del modelo EFQM en la Generalit Valenciana", Revista Valenciana d'Estudis Autonòmics, nº 34, págs. 99-119.

GÓMEZ, P.; y SATURNO, P. J. (1997): “La calidad en atención primaria de salud según sus diferentes protagonistas", en I Congreso Regional de Calidad Asistencial. Murcia, 27 y 28 de noviembre de 1997, Murcia [<http://www. murciasalud.es/archivo.php?id=136568〉].

GRÖNROOS, C. (1994): Marketing y gestión de servicios, Madrid, Díaz de Santos.

- (1988): "Service quality: The six criteria of good service quality", Review of Business, $\mathrm{n}^{\circ}-3$.

GUMMERSON, E. (1987): Quality-The Ericsson Approach, Estocolmo, Ericsson.

HOFFMAN, D.; y BATESON, J. (2002): Fundamentos de marketing de servicios, Madrid, McGraw Hill.

IGAMI, M. P. (2005): “El uso del SERVQUAL en la verificación de la calidad de los servicios de unidades de información: el caso de la biblioteca del IPEN", Revista Interamericana de Bibliotecología, vol. 28, noำ 2, págs. 177-191 [<http://hdl.handle. net/10760/9095'].

PÉREZ MEYANO, V. (2007) “El INSS: una apuesta por la calidad". Comunicación presentada en la XXI Reunión de Referentes de Progreso 2007. Madrid, 18 de octubre de 2007 - Barcelona, 25 de octubre de 2007 [<http://www.qualitat.org/ cgi-bin/view/97cpvpm.htmls].

KOELEMEIJER, K.; ROEST, H.; y VERHALLEN, T. (1993): “An integrative framework of perceived service quality and its relations to satisfaction/ insatisfaction, attitude and repurchase intention. A multilevel approach", en European Marketing Academy (EMAC). Proceedings, Barcelona, ESADE, págs. 25-28 [ [http://arno. uvt.nl/show.cgi?fid=10852>].
MARTÍNEZ DEL OLMO, M. T. (1994): "La calidad de los servicios sanitarios y la participación de los usuarios/as", en IX Jornadas de Salud Pública y Administración Sanitaria, Granada, Escuela Andaluza de Salud Pública, pág. 81.

MCELWEE, G.; y REDMAN, T. (1993): “Upward appraisal in practice: An illustrative example using the QUALED scale", Education and Training, vol. $35, \mathrm{n}-2$.

MEDINA RUIZ, E. (2011): Evaluación de la calidad percibida en servicios sociales, Murcia, Diego Marín Editor.

MEDINA TORNERO, M. E. (2000): Evaluación de la calidad asistencial del servicio de ayuda a domicilio, Murcia, Universidad de Murcia.

- (1997a): "La evaluación de la calidad de los servicios sociales", en II Congreso de Psicología Profesional, Murcia, Colegio Oficial de Psicólogos.

- (1997b): "Utilidad de la evaluación en los servicios sociales comunitarios", Apuntes de Psicología, no 49-50, págs. 127-151.

- (1996): "Evaluación de la calidad asistencial en servicios sociales", Intervención Psicosocial, vol. 5 , no 14, págs. 23-42 [rhttp://www.copmadrid. org/webcopm/publicaciones/social/1996/ vol2/arti2.htm〉].

MEDINA TORNERO, M. E.; y MEDINA RUIZ, E. (2011): La calidad en las leyes de servicios sociales autonómicas. Estudio presentado en el $X$ Congreso de Organizaciones de Mayores, Albacete, 12-14 de abril de 2011.

MORALES SÁNCHEZ, V. (2003): Evaluación psicosocial de la calidad en servicios municipales deportivos: aportaciones desde el análisis de la variabilidad, Málaga, Spicum.

MORALES, M.; y CALDERÓN, L. F. (2005): “Desarrollo de un instrumento para medir la calidad de servicio en cursos y programas de educación para ejecutivos", Revista de Empresa, vol. 13, nํㅜ 1, págs. 30-41.

MORRIS, S. (1992): Citizens and Local Democracy: Encouraging and Managing Complaints, Luton, Local Government Management Board.

MOYA VAQUERO, E. (2000): "Herramientas para gestionar la calidad de un centro de servicios sociales", Revista de Servicios Sociales y Política Social, nํ- 49, págs. 85-90.

NGUYEN, N. (1991): “Un modèle explicatif de l'évaluation de la qualité d'un service: une étude empirique", Recherche et Applications en Marketing, vol. 6, nํㅡ 2, págs. 83-98.

PALLARES, L.; y GARCÍA, M. J. (1996): Guía práctica para la evaluación de la calidad en la atención enfermera, Madrid, Olalla Ediciones.

PARASURAMAN, A.; ZEITHAML, V.; y BERRY, L. (1994): "Reassessment of expectations as a comparison standard in measuring service quality: Implications for further research", Journal of Marketing, $\mathrm{n}^{0}$ 58, págs. 111-124 ['http://goo.gl/DsrVP)].

- (1993): Calidad total en la gestión de servicio, Madrid, Díaz de Santos. 
- (1988): "SERVQUAL: A multiple scale for measuring consumer perceptions of service quality", Journal of Retailing, n- 64, págs. 12-40.

- (1985): "A conceptual model of service quality and its implications for future research", Journal of Retailing, nํ49, págs. 44-60 [rhttp://areas. kenan-flagler.unc.edu/Marketing/FacultyStaff/ zeithaml/Selected\%20Publications/A\%20 Conceptual\%2oModel\%20of\%20Service $\% 20$ Quality\%20and\%20Its\%2olmplications\%20 for $\% 20$ Future\%20Research.pdf $>$.

PARREÑO, A. (2007): Retos para la atención primaria de los servicios sociales en el marco de la Ley de Promoción de la Autonomía Personal [rhttp:// www2. Mancohortasud.es/otrosproyectos/ Ponencia_Angel_Parreño.pdfı].

PÉREZ MEYANO, V. (2007) “El INSS: una apuesta por la calidad”. Comunicación presentada en la XXI Reunión de Referentes de Progreso 2007. Madrid, 18 de octubre de 2007 - Barcelona, 25 de octubre de 2007 [<http://www.qualitat.org/ cgi-bin/view/97cpvpm.htmlı?].

PÉREZ MOROTE, R. (2001): “El análisis de las demandas ciudadanas y la consecución de servicios públicos municipales de calidad total: especial referencia al servicio de recogida de residuos sólidos urbanos", Análisis Local, nํ34, págs. 45-56.

REAL ACADEMIA ESPAÑOLA (1992): Diccionario de la Real Academia Española, Madrid, Real Academia Española.

SACANELL, E. (2003): "Calidad y servicios sociales: la experiencia del Ayuntamiento de Getxo", en SETIÉN, Ma L. L; y SACANELL, E. (coords.), La calidad de los servicios sociales: conceptos y experiencias, Valencia, Tirant lo Blanc, págs. 297-316.
SÁNCHEZ AGUADO, A. (2011): "La calidad en la gestión de los servicios sociales municipales: algunas experiencias de INTRESS", TS Nova. Trabajo Social y Servicios Sociales, no 3 , págs. 49-54.

SÁNCHEZ PÉREZ, M. C. (2010): “Calidad de satisfacción de usuarios: una investigación para la mejora de los servicios sociales", en RODRÍGUEZ MONGE, Á.; y RAYA LOZANO, E. E. (coords.) Una Europa social y plural. Actas del VII Congreso Estatal de Escuelas Universitarias de Trabajo Social, Granada, Universidad de Granada, págs. 1.4571.467.

SATURNO, P. J. (1991): "Métodos de evaluación de la calidad asistencial (prólogo a la edición española)", en NUTTING, P. et al., Métodos de evaluación de la calidad en atención primaria: guía para clínicos, Barcelona, SG Editores.

SENLLE, A. (1996): Calidad total en los servicios y en la administración pública, Barcelona, Gestión 200.

SUÑOL, R. (1987): “El estudio de la opinión del usuario y su aplicación en los programas de control de calidad”, Control de Calidad Asistencial, no 2.

VILA, I.; SÁNCHEZ, C.; y MANASSERO, Ma. . A. (2009) "Satisfacción percibida de los usuarios de las instalaciones deportivas municipales de Palma de Mallorca", Revista Iberoamericana de Psicología del Ejercicio y el Deporte, vol. 4, no - 1, págs. 59-74 [<http://www.webs.ulpgc.es/riped/ docs/20090104.pdfs].

VILLEGAS, M. M.; y ROSA, I. M. (2003): "La calidad asistencial: concepto y medida”, Dirección y Organización, no 29 [shttp://www.revistadyo. com/index.php/dyo/article/view/142/142>].

VUORI, H. (1998): El control de calidad en los servicios sanitarios, Madrid, Masson. 